1. Status of this Memo

This document is being distributed to members of the Internet community in order to solicit their reactions to the proposals contained in it. While the issues discussed may not be directly relevant to the research problems of the Internet, they may be interesting to a number of researchers and implementors.

This memo defines a simple application-layer protocol by which management information for a gateway may be inspected or altered by logically remote users.

This proposal is intended only as an interim response to immediate gateway monitoring needs while work on more elaborate and robust designs proceeds with the care and deliberation appropriate to that task. Accordingly, long term use of the mechanisms described here should be seriously questioned as more comprehensive proposals emerge in the future. Distribution of this memo is unlimited.

2. Protocol Design Strategy

The proposed protocol is shaped in large part by the desire to minimize the number and complexity of management functions realized by the gateway itself. This goal is attractive in at least four respects:

(1) The development cost for gateway software necessary to support the protocol is accordingly reduced.

(2) The degree of management function that is remotely supported is accordingly increased, thereby admitting fullest use of internet resources in the management task. 
(3) The degree of management function that is remotely supported is accordingly increased, thereby imposing the fewest possible restrictions on the form and sophistication of management tools.

(4) A simplified set of management functions is easily understood and used by developers of gateway management tools.

A second design goal is that the functional paradigm for monitoring and control be sufficiently extensible to accommodate additional, possibly unanticipated aspects of gateway operation.

A third goal is that the design be, as much as possible, independent of the architecture and mechanisms of particular hosts or particular gateways.

Consistent with the foregoing design goals are a number of decisions regarding the overall form of the protocol design.

One such decision is to model all gateway management functions as alterations or inspections of various parameter values. By this model, a protocol entity on a logically remote host (possibly the gateway itself) interacts with a protocol entity resident on the gateway in order to alter or retrieve named portions (variables) of the gateway state. This design decision has at least two positive consequences:

(1) It has the effect of limiting the number of essential management functions realized by the gateway to two: one operation to assign a value to a specified configuration parameter and another to retrieve such a value.

(2) A second effect of this decision is to avoid introducing into the protocol definition support for imperative management commands: the number of such commands is in practice ever-increasing, and the semantics of such commands are in general arbitrarily complex.

The exclusion of imperative commands from the set of explicitly supported management functions is unlikely to preclude any desirable gateway management operation. Currently, most gateway commands are requests either to set the value of some gateway parameter or to retrieve such a value, and the function of the few imperative commands currently supported is easily accommodated in an asynchronous mode by this management model. In this scheme, an imperative command might be realized as the setting of a parameter value that subsequently triggers the desired action. 
A second design decision is to realize any needed authentication functionality in a distinct protocol layer that provides services to the monitoring protocol itself. The most important benefit of this decision is a reduction in the complexity of the individual protocol layers - thereby easing the task of implementation.

Consistent with this layered design strategy is a third design decision that the identity of an application protocol entity is known to its peers only by the services of the underlying authentication protocol. Implicit in this decision is a model of access control by which access to variables of a gateway configuration is managed in terms of the association between application entities and sessions of the authentication protocol. Thus, multi-level access to gateway variables is supported by multiple instances of the application protocol entity, each of which is characterized by:

(1) the set of gateway variables known to said entity,

(2) the mode of access (READ-ONLY or READ-WRITE) afforded to said set of variables, and

(3) the authentication protocol session to which belong the messages sent and received by said entity.

A fourth design decision is to adopt the conventions of the CCITT X.409 recommendation [1] for representing the information exchanged between protocol entities. One cost of this decision is a modest increase in the complexity of the protocol implementation. One benefit of this decision is that protocol data are represented on the network in a machine-independent, widely understood, and widely accepted form. A second benefit of this decision is that the form of the protocol messages may be concisely and understandably described in the X.409 language defined for such purposes.

A fifth design decision, consistent with the goal of minimizing gateway complexity, is that the variables manipulated by the protocol assume only integer or octet string type values.

A sixth design decision, also consistent with the goal of minimizing gateway complexity, is that the exchange of protocol messages requires only an unreliable datagram transport, and, furthermore, that every protocol message is entirely and independently representable by a single transport datagram. While this document specifies the exchange of protocol messages via the UDP protocol [2], the design proposed here is in general suitable for use with a wide variety of transport mechanisms. 
A seventh design decision, consistent with the goals of simplicity and extensibility, is that the variables manipulated by the protocol are named by octet string values. While this decision departs from the architectural traditions of the Internet whereby objects are identified by assigned integer values, the naming of variables by octet strings affords at least two valuable benefits. Because the set of octet string values constitutes a variable name space that, as convenient, manifests either flat or hierarchical structure,

(1) a single, simple mechanism can provide both random access to individual variables and sequential access to semantically related groups of variables, and

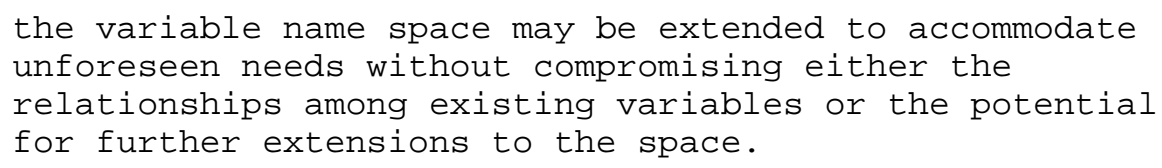

An eighth design decision is to minimize the number of unsolicited messages required by the protocol definition. This decision is consistent with the goal of simplicity and motivated by the desire to retain maximal control over the amount of traffic generated by the network management function - even at the expense of additional protocol overhead. The strategy implicit in this decision is that the monitoring of network state at any significant level of detail is accomplished primarily by polling for appropriate information on the part of the monitoring center. In this context, the definition of unsolicited messages in the protocol is confined to those strictly necessary to properly guide a monitoring center regarding the timing and focus of its polling.

3. The Gateway Monitoring Protocol

The gateway monitoring protocol is an application protocol by which the variables of a gateway's configuration may be inspected or altered.

Communication among application protocol entities is by the exchange of protocol messages using the services of the authentication protocol described elsewhere in this document. Each such message is entirely and independently represented by a single message of the underlying authentication protocol. An implementation of this protocol need not accept protocol messages whose length exceeds 484 octets.

The form and function of the four message types recognized by a protocol entity is described below. The type of a given protocol message is indicated by the value of the implicit type tag for the 
data structure that is represented by said message according to the conventions of the CCITT X.409 recommendation.

3.1. The Get Request Message Type

The form of a message of Get Request type is described below in the language defined in the CCITT X.409 recommendation:

var_value_type

$::=\quad$ CHOICE \{

INTEGER, OCTET STRING

\}

var_name_type

$:=$

OCTET STRING

var_op_type

$::=$

SEQUENCE \{

var_name

var_name_type,

var_value

var_value_type

\}

var_op_list_type

$::=$

SEQUENCE OF var_op_type

error_status_type

$::=$

INTEGER \{

gmp_err_noerror

(0),

gmp_err_too_big

(1),

gmp_err_nix_name

(2),

gmp_err_bad_value

(3)

\}

error_index_typ

$::=$

INTEGER

request_id_type

$::=$

INTEGER

get_req_message_type

$::=$

[ APPLICATION 1 ] IMPLICIT

SEQUENCE \{

request_id

error_status

error_index

var_op_list

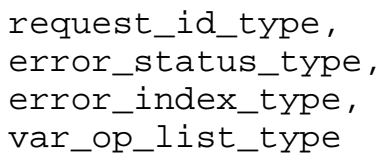


Upon receipt of a message of this type, the receiving entity responds according to any applicable rule in the list below:

(1) If, for some var_op_type component of the received message, the value of the var_name field does not lexicographically precede the name of some variable known to the receiving entity, then the receiving entity sends to the originator of the received message a message of identical form except that the indicated message type is Get Response, the value of the error_status field is gmp_err_nix_name, and the value of the error_index field is the unit-based index of said var_op_type component in the received message.

(2) If the size of the Get Response type message generated as described below would exceed the size of the largest message for which the protocol definition requires acceptance, then the receiving entity sends to the originator of the received message a message of identical form except that the indicated message type is Get Response, the value of the error_status field is gmp_err_too_big, and the value of the error_index field is zero.

If none of the foregoing rules apply, then the receiving entity sends to the originator of the received message a Get Response type message such that, for each var_op_type component of the received message, a corresponding component of the generated message represents the name and value of that variable whose name is, in the lexicographical ordering of the names of all variables known to the receiving entity together with the value of the var_name field of the given component, the immediate successor to that value. The value of the error_status field of the generated message is gmp_err_noerror and the value of the error_index field is zero. The value of the request_id field of the generated message is that for the received message.

Messages of the Get Request type are generated by a protocol entity only at the request of the application user.

\subsection{The Get Response Message Type}

The form of messages of this type is identical to that of Get Request type messages except for the indication of message type. In the CCITT X.409 language,

get_rsp_message_type $::=\quad[$ APPLICATION 2 ] IMPLICIT

\section{SEQUENCE \{}




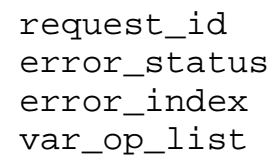

3.3. The Trap Request Message Type

The form of a message of this type is described below in the language defined in the CCITT X.409 recommendation:

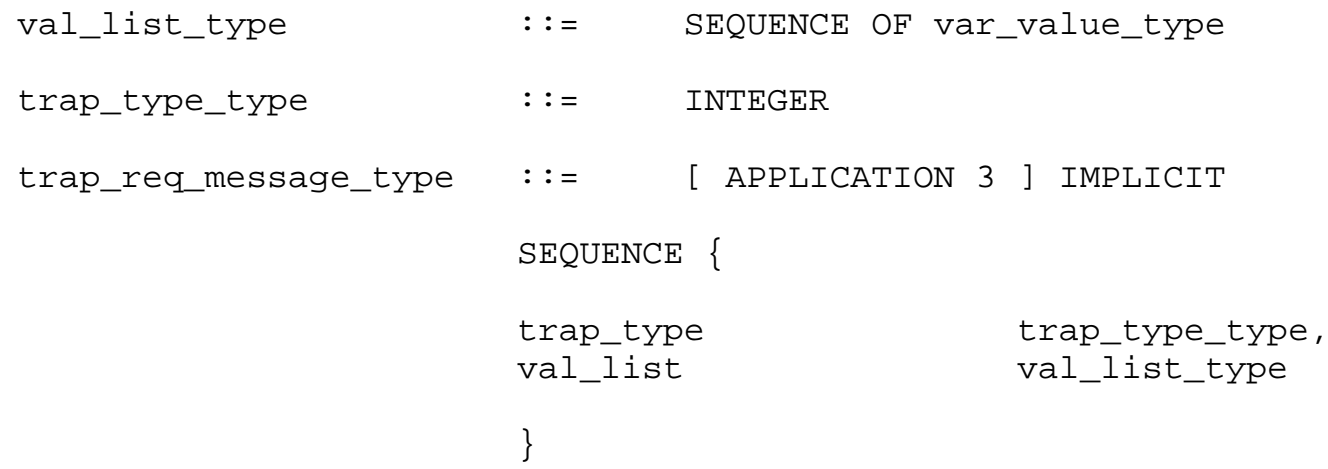


field is 0, signifies that the sending protocol entity is reinitializing itself such that the gateway configuration or the protocol entity implementation may be altered.

\subsubsection{The Warm Start Trap Type}

A Trap Request type message for which the value of the trap_type field is 1, signifies that the sending protocol entity is reinitializing itself such that neither the gateway configuration nor the protocol entity implementation is altered.

\subsubsection{The Link Failure Trap Type}

A Trap Request type message for which the value of the trap_type field is 2, signifies that the sending protocol entity recognizes a failure in one of the communication links represented in the gateway configuration.

\subsubsection{The Authentication Failure Trap Type}

A Trap Request type message for which the value of the trap_type field is 3, signifies that the sending protocol entity is the addressee of a protocol message that is not properly authenticated.

3.3.5. The EGP Neighbor Loss Trap Type

A Trap Request type message for which the value of the trap_type field is 4, signifies that an EGP neighbor for whom the sending protocol entity was an EGP peer has been marked down and the peer relationship no longer obtains.

3.4. The Set Request Message Type

The form of messages of this type is identical to that of Get Request type messages except for the indication of message type. In the CCITT X.409 language:

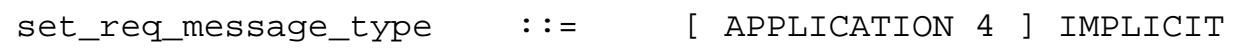


Upon receipt of a message of this type, the receiving entity responds according to any applicable rule in the list below:

(1) If, for some var_op_type component of the received message, the value of the var_name field names no variable known to the receiving entity, then the receiving entity sends to the originator of the received message a message of identical form except that the indicated message type is Get Response, the value of the error_status field is gmp_err_nix_name, and the value of the error_index field is the unit-based index of said var_op_type component in the received message.

(2) If, for some var_op_type component of the received message, the contents of the var_value field does not, according to the CCITT X.409 recommendation, manifest a type, length, and value that is consistent with that required for the variable named by the value of the var_name field, then the receiving entity sends to the originator of the received message a message of identical form except that the indicated message type is Get Response, the value of the error_status field is gmp_err_bad_value, and the value of the error_index field is the unit-based index of said var_op_type component in the received message.

(3) If the size of the Get Response type message generated as described below would exceed the size of the largest message for which the protocol definition requires acceptance, then the receiving entity sends to the originator of the received message a message of identical form except that the indicated message type is Get Response, the value of the error_status field is gmp_err_too_big, and the value of the error_index field is zero.

If none of the foregoing rules apply, then for each var_op_type component of the received message, according to the sequence of such components represented by said message, the value represented by the var_value field of the given component is assigned to the variable named by the value of the var_name field of that component. The receiving entity sends to the originator of the received message a message of identical form except that the indicated message type is Get Response, the value of the error_status field is gmp_err_noerror, and the value of the error_index field is zero.

Messages of the set Request type are generated by a protocol entity only at the request of the application user.

Recognition and processing of set Request type frames is not required by the protocol definition. 


\section{The Authentication Protocol}

The authentication protocol is a session-layer protocol by which messages specified by a protocol user are selectively delivered to other protocol users. The protocol definition precludes delivery to a protocol user of any user message for which the protocol representation lacks a specified "authentic" form.

Communication among authentication protocol entities is accomplished by the exchange of protocol messages, each of which is entirely and independently represented by a single UDP datagram. An authentication protocol entity responds to protocol messages received at UDP port 153 on the host with which it is associated.

A half-session of the authentication protocol is, for any ordered pair of protocol users, the set of messages sent from the first user of the pair to the second user of said pair. A session of the authentication protocol is defined to be union of two complementary half-sessions of the protocol - that is, the set of messages exchanged between a given pair of protocol users. Associated with each protocol half-session is a triplet of functions:

(1) The authentication function for a given half-session is a boolean-valued function that characterizes the set of authentication protocol messages that are of acceptable, authentic form with respect to the set of all possible authentication protocol messages.

(2) The message interpretation function for a given halfsession is a mapping from the set of authentication protocol messages accepted by the authentication function for said half-session to the set of all possible user messages.

(3) The message representation function for a given halfsession is a mapping that is the inverse of the message interpretation function for said half-session.

The association between half-sessions of the authentication protocol and triplets of functions is not defined in this document.

The form and function of the single message type recognized by a protocol entity is described below. The type of a given protocol message is indicated by the value of the implicit type tag for the data structure that is represented by said message according to the conventions of the CCITT X.409 recommendation. 


\subsection{The Data Request Message Type}

Messages of this type are represented by a sequence of fields whose form and interpretation are described below.

\subsubsection{The Message Length Field}

The Message Length field of a given Data Request message represents the length of said message as an unsigned, 16-bit, binary integer. This value is encoded such that more significant bits precede less significant bits in the order of transmission and includes the length of the Message Length field itself.

\subsubsection{The Session ID Length Field}

The Session ID Length field of a given Data Request message represents the length, in octets, of the Session ID field of said message. This value is encoded as an unsigned, 8-bit, binary integer.

\subsubsection{The Session ID Field}

The Session ID field of a given Data Request message represents the name of the protocol session to which said message belongs. The value of this field is encoded as asequence of octets whose length is the value of the Session ID Length field for said message.

\subsubsection{The User Data Field}

The User Data field of a given Data Request message represents a message being passed from one protocol user to another. The value of this field is encoded according to conventions implicit in the message representation function for the appropriate half of the protocol session named by the value of the session ID field for said message.

Upon receipt of a Data Request type message, the receiving authentication protocol entity verifies the form of said message by application of the authentication function associated with its half of the session named by the value of the Session ID field in the received message. If the form of the received message is accepted as "authentic" by said function, then the user message computed by the application of the message interpretation function for said halfsession to the value of the User Data field of the received message is presented to the protocol user together with an indication of the protocol session to which the received message belongs. 
Otherwise, the message is discarded and an indication of the receipt of an unauthenticated message is presented to the protocol user.

A message of this type is generated only at the request of the protocol user to communicate a message to another user of the protocol. Such a request specifies the user message to be sent as well as the session of the authentication protocol to which said user message belongs. The value of the Session ID field of the generated message is the name of the session specified in the user request. The value of the User Data field of the generated message is computed by applying the message representation function for the appropriate half of the specified session to the specified user message.

5. Variable Names

The variables retrieved or manipulated by the application protocol are named by octet string values. Such values are represented in this document in two ways:

(1) A variable name octet string may be represented numerically by a sequence of hexadecimal numbers, each of which denotes the value of the corresponding octet in said string.

(2) A variable name octet string may be represented symbolically by a character string whose form reflects the sequence of octets in said name while at the same time suggesting to a human reader the semantics of the named variable.

Variable name octet strings are represented symbolically according to the following two rules:

(1) The symbolic character string representation of the variable name of zero length is the character string of zero length.

(2) The symbolic character string representation of a variable name of non-zero length $\mathrm{n}$ is the concatenation of the symbolic character string representation of the variable name formed by the first ( $n-1)$ octets of the given name together with the underscore character ("-") and a character string that does not include the underscore character, such that the resulting character string is unique among the symbolic character string representations for all variable names of length $\mathrm{n}$. 
Thus, for example, the variable names represented numerically as:

$$
\begin{array}{llllll}
01 & 01 & 01, & & \\
01 & 01 & 02, & & & \\
01 & 02 & 01, & & & \\
01 & 03 & 01 & 03 & 01, & \\
01 & 03 & 01 & 03 & 02, & \\
01 & 03 & 01 & 04 & 01, & \text { and } \\
01 & 03 & 01 & 04 & 02 &
\end{array}
$$

might be represented symbolically by the character strings:

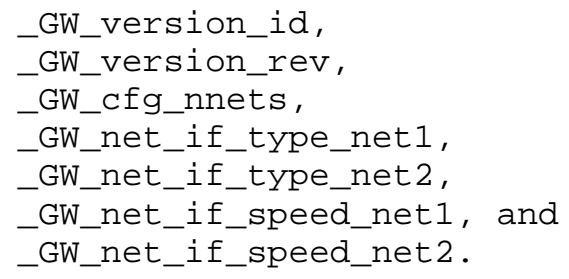

All variable names are terminated by an implementation specific octet string of non-zero length. Thus, a complete variable name is not specified for any of the variables defined in this document. Rather, for each defined variable, some prefix portion of its name is specified, with the understanding that the rightmost portion of its name is specific to the protocol implementation.

Fullest exploitation of the semantics of the Get Request type message requires that names for related variables be chosen so as to be contiguous in the lexicographic ordering of all variable names recognized by an application protocol entity. This principle is observed in the naming of variables currently defined by this document, and it should be observed as well for variables defined by subsequent revisions of this document and for variables introduced by particular implementations of the protocol.

A particular implementation of a protocol entity may present variables in addition to those defined by this document, provided that in no case will an implementation-specific variable be presented as having a name identical to that for one of the variables defined here. By convention, the names of variables specific to a particular implementation share a common prefix that distinguishes said variables from those defined in this document and from those that may be presented by other implementations of an application protocol entity. For example, variables specific to an implementation of this protocol in version 1.3 of the squeaky gateway product of the Swinging Gateway company might have the names represented by: 
$01 \mathrm{FF} 01011301$,

01 FF $0101 \quad 13 \quad 02$, and

01 FF 01011303 ,

for which the corresponding symbolic representations might be:

$$
\begin{aligned}
& \text { _GW_impl_Swinging_Squeaky_v1.3_variableA, } \\
& \text { _GW_impl_Swinging_Squeaky_v1.3_variableB, and } \\
& \text { _GW_impl_Swinging_Squeaky_v1.3_variableC. }
\end{aligned}
$$

The names and semantics of implementation-specific variables are not otherwise defined by this document, although implementors are encouraged to publish such definitions either as appendices to this document or by other appropriate means.

Variable names of which the initial portion is represented numerically as 02 and symbolically as "_HOST" are reserved for future use. Variable names of which the initial portion is represented numerically as 03 and symbolically as "_TS" are similarly reserved.

6. Required Variables

To the extent that the information represented by a variable defined in this section is also represented internally by a gateway for which this protocol is realized, access to that variable must be afforded by at least one application protocol entity associated with said gateway.

6.1. The _GW_version_id Variable

The variable such that the initial portion of its name is represented symbolically as "_GW_version_id" and numerically as:

$$
010101
$$

has an octet string value that identifies the protocol entity implementation (e.g., "ACME Packet-Whiz Model II").

6.2. The _GW_version_rev Variable

The variable such that the initial portion of its name is represented symbolically as "_GW_version_rev" and numerically as:

\section{2}

has an integer value that identifies the revision level of the entity implementation. The encoding of the revision level as an integer 
value is implementation-specific.

6.3. The _GW_cfg_nnets Variable

The variable such that the initial portion of its name is represented symbolically as "_GW_cfg_nnets" and numerically as:

010201

has an integer value that represents the number of logical network interfaces afforded by the configuration of the gateway.

\subsection{Network Interface Variables}

This section describes a related set of variables that represent attributes of the logical network interfaces afforded by the gateway configuration. Each such network interface is uniquely identified by an octet string. The convention by which names are assigned to the network interfaces of a gateway is implementation-specific.

6.4.1. The _GW_net_if_type Variable Class

A variable such that the initial portion of its name is represented symbolically as "_GW_net_if_type" and numerically as:

\section{$\begin{array}{llll}01 & 03 & 01 & 03\end{array}$}

has an integer value that represents the type of the network interface identified by the remainder of the name for said variable. The value of a variable of this class represents network type according to the conventions described in Appendix 1.

6.4.2. The _GW_net_if_speed Variable Class

A variable such that the initial portion of its name is represented symbolically as "_GW_net_if_speed" and numerically as:

01030104

has an integer value that represents the estimated nominal bandwidth in bits per second of the network interface identified by the remainder of the name for said variable.

6.4.3. The _GW_net_if_in_pkts Variable Class

A variable such that the initial portion of its name is represented symbolically as "_GW_net_if_in_pkts" and numerically as: 
0103010101

has an integer value that represents the number of packets received by the gateway over the network interface identified by the remainder of the name for said variable.

6.4.4. The _GW_net_if_out_pkts Variable Class

A variable such that the initial portion of its name is represented symbolically as "_GW_net_if_out_pkts" and numerically as:

$$
0103010201
$$

has an integer value that represents the number of packets

transmitted by the gateway over the network interface identified by the remainder of the name for said variable.

6.4.5. The _GW_net_if_in_bytes Variable Class

A variable such that the initial portion of its name is represented symbolically as "_GW_net_if_in_bytes" and numerically as:

$$
0103010102
$$

has an integer value that represents the number of octets received by the gateway over the network interface identified by the remainder of the name for said variable.

6.4.6. The _GW_net_if_out_bytes Variable Class

A variable such that the initial portion of its name is represented symbolically as "_GW_net_if_out_bytes" and numerically as:

$01030102 \quad 02$

has an integer value that represents the number of octets transmitted by the gateway over the network interface identified by the remainder of the name for said variable.

6.4.7. The _GW_net_if_in_errors Variable Class

A variable such that the initial portion of its name is represented symbolically as "_GW_net_if_in_errors" and numerically as:

\section{3}

has an integer value that represents the number of reception errors encountered by the gateway on the network interface identified by the 
remainder of the name for said variable. The definition of a reception error is implementation-specific and may vary according to network type.

6.4.8. The _GW_net_if_out_errors Variable Class

A variable such that the initial portion of its name is represented symbolically as "_GW_net_if_out_errors" and numerically as:

$$
0103 \quad 0102 \quad 03
$$

has an integer value that represents the number of transmission errors encountered by the gateway on the network interface identified by the remainder of the name for said variable. The definition of a transmission error is implementation-specific and may vary according to network type.

6.4.9. The _GW_net_if_status Variable Class

A variable such that the initial portion of its name is represented symbolically as "_GW_net_if_status" and numerically as:

$$
01030105
$$

has an integer value that represents the current status of the network interface identified by the remainder of the name for said variable. Network status is represented according to the conventions described in Appendix 2.

\subsection{Internet Protocol Variables}

This section describes variables that represent information related to protocols and mechanisms of the Internet Protocol (IP) family [3].

\subsubsection{Protocol Address Variable Classes}

This section describes a related set of variables that represent attributes of the the IP interfaces presented by a gateway on the various networks to which it is attached. Each such protocol interface is uniquely identified by an octet string. The convention by which names are assigned to the protocol interfaces for a gateway is implementation-specific.

6.5.1.1. The _GW_pr_in_addr_value Variable Class

A variable such that the initial portion of its name is represented symbolically as "_GW_pr_in_addr_value" and numerically as: 
0104010101

has an octet string value that literally represents the 32-bit Internet address for the IP interface identified by the remainder of the name for said variable.

6.5.1.2. The _GW_pr_in_addr_scope Variable Class

A variable such that the initial portion of its name is represented symbolically as "_GW_pr_in_addr_scope" and numerically as:

0104010102

has an octet string value that names the network interface with which the IP interface identified by the remainder of the name for said variable is associated.

\subsubsection{Exterior Gateway Protocol (EGP) Variables}

This section describes variables that represent information related to protocols and mechanisms of the EGP protocol [4].

6.5.2.1. The _GW_pr_in_egp_core Variable

A variable such that the initial portion of its name is represented symbolically as "_GW_pr_in_egp_core" and numerically as:

\section{1}

has an integer value that characterizes the associated gateway with respect to the set of INTERNET core gateways. A nonzero value indicates that the associated gateway is part of the INTERNET core.

6.5.2.2. The _GW_pr_in_egp_as Variable Class

A variable such that the initial portion of its name is represented symbolically as "_GW_pr_in_egp_as" and numerically as:

$$
\begin{array}{lllll}
01 & 04 & 01 & 03 & 02
\end{array}
$$

has an integer value that literally identifies an Autonomous system to which this gateway belongs.

\subsubsection{The EGP Neighbor Variable Classes}

This section describes a related set of variables that represent attributes of "neighbors" with which the gateway may be associated by EGP. Each such EGP neighbor is uniquely identified by an octet 
string. The convention by which names are assigned to EGP neighbors of a gateway is implementation-specific.

6.5.2.3.1. The _GW_pr_in_egp_neighbor_addr Variable Class

A variable such that the initial portion of its name is represented symbolically as "_GW_pr_in_egp_neighbor_addr" and numerically as:

$01040103 \quad 03 \quad 01$

has an octet string value that literally represents the 32-bit Internet address for the EGP neighbor identified by the remainder of the name for said variable.

6.5.2.3.2. The_GW_pr_in_egp_neighbor_state Variable Class

A variable such that the initial portion of its name is represented symbolically as "_GW_pr_in_egp_neighbor_state" and numerically as:

$$
01040103 \quad 03 \quad 02
$$

has an octet string value that represents the EGP protocol state of the gateway with respect to the EGP neighbor identified by the remainder of the name for said variable. The meaningful values for such a variable are: "IDLE," "ACQUISITION, " "DOWN, " "UP, " and "CEASE."

6.5.2.4. The _GW_pr_in_egp_errors Variable

The variable such that the initial portion of its name is represented symbolically as "_GW_pr_in_egp_errors" and numerically as:

$01040103 \quad 05$

has an integer value that represents the number of EGP protocol errors.

\subsubsection{Routing Variable Classes}

This section describes a related set of variables that represent attributes of the the IP routes by which a gateway directs packets to various destinations on the Internet. Each such route is uniquely identified by an octet string that is the concatenation of the literal 32-bit value of the Internet address for the destination of said route together with an implementation-specific octet string. The convention by which names are assigned to the Internet routes for a gateway is in all other respects implementation-specific. 
6.5.3.1. The _GW_pr_in_rt_gateway Variable Class

A variable such that the initial portion of its name is represented symbolically as "_GW_pr_in_rt_gateway" and numerically as:

$$
0104010201
$$

has an octet string value that literally represents the 32-bit Internet address of the next gateway to which traffic is directed by the route identified by the remainder of the name for said variable.

6.5.3.2. The _GW_pr_in_rt_type Variable Class

A variable such that the initial portion of its name is represented symbolically as "_GW_pr_in_rt_type" and numerically as:

$010401 \quad 02 \quad 02$

has an integer value that represents the type of the route identified by the remainder of the name for said variable. Route types are identified according to the conventions described in Appendix 3.

6.5.3.3. The _GW_pr_in_rt_how-learned Variable Class

A variable such that the initial portion of its name is represented symbolically as "_GW_pr_in_rt_how-learned" and numerically as:

$$
0104010203
$$

has an octet string value that represents the source of the information from which the route identified by the remainder of the name for said variable is generated. The meaningful values of such a variable are: "STATIC," "EGP," and "RIP."

6.5.3.4. The _GW_pr_in_rt_metric0 Variable Class

A variable such that the initial portion of its name is represented symbolically as "_GW_pr_in_rt_metric0" and numerically as:

$$
01040102 \quad 04
$$

has an integer value that represents the quality (in terms of cost, distance from the ultimate destination, or other metric) of the route identified by the remainder of the name for said variable.

6.5.3.5. The _GW_pr_in_rt_metric1 Variable Class

A variable such that the initial portion of its name is represented 
symbolically as "_GW_pr_in_rt_metricl" and numerically as:

$$
01040102 \quad 05
$$

has an integer value that represents the quality (in terms of cost, distance from the ultimate destination, or other metric) of the route identified by the remainder of the name for said variable.

6.6. DECnet Protocol Variables

This section describes variables that represent information related to protocols and mechanisms of the DEC Digital Network Architecture. DEC and DECnet are registered trademarks of Digital Equipment Corporation.

\subsection{XNS Protocol Variables}

This section describes variables that represent information related to protocols and mechanisms of the Xerox Network System. Xerox Network System and XNS are registered trademarks of the XEROX Corporation.

7. Implementation-Specific Variables

Additional variables that may be presented for inspection or manipulation by particular protocol entity implementations are described in Appendices to this document.

8. References

[1] CCITT, "Message Handling Systems: Presentation Transfer Syntax and Notation", Recommendation X.409, 1984.

[2] Postel, J., "User Datagram Protocol", RFC-768, USC/Information Sciences Institute, August 1980.

[3] Postel, J., "Internet Protocol", RFC-760, USC/Information Sciences Institute, January 1980.

[4] Rosen, E., "Exterior Gateway Protocol", RFC-827, Bolt Beranek and Newman, October 1982 .

9. Appendix 1: Network Type Representation

Numeric representations for various types of networks are presented below: 


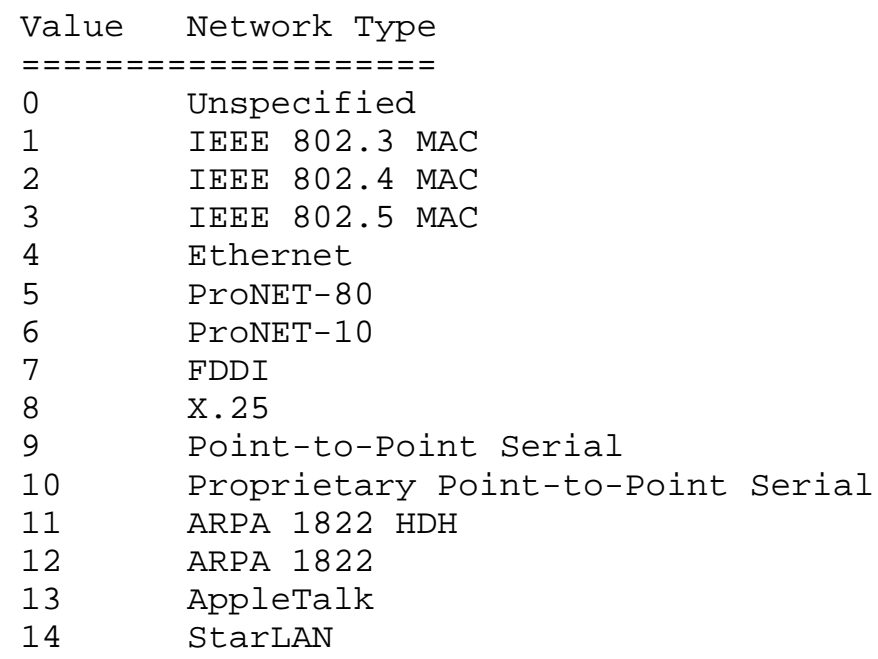

10. Appendix 2: Network Status Representation

Numeric representations for network status are presented below.

$\begin{array}{ll}\text { Value } & \text { Network Status } \\ ================== & ==0 \\ 0 & \text { Interface Operating Normally } \\ 1 & \text { Interface Not Present } \\ 2 & \text { Interface Disabled } \\ 3 & \text { Interface Down } \\ 4 & \text { Interface Attempting Link }\end{array}$

11. Appendix 3: Route Type Representation

Numeric representations for route types are presented below.

$\begin{array}{ll}\begin{array}{l}\text { Value } \\ =======\end{array} & \begin{array}{l}\text { Route } \\ \text { Type }\end{array}===== \\ 0 & \text { Route to Nowhere }-- \text { ignored } \\ 1 & \text { Route to Directly Connected Network } \\ 2 & \text { Route to a Remote Host } \\ 3 & \text { Route to a Remote Network } \\ 4 & \text { Route to a Sub-Network }\end{array}$

12. Appendix 4: Initial Implementation Strategy

The initial objective of implementing the protocol specified in this document is to provide a mechanism for monitoring Internet gateways. While the protocol design makes some provision for gateway management 
functions as well, this aspect of the design is not fully developed and needs further refinement before a generally useful implementation could be produced. Accordingly, initial implementations will not generate or respond to the optional set Request message type.

The protocol defined here may be subsequently refined based upon experience with early implementations or upon further study of the problem of gateway management. Moreover, it may be superceded by other proposals in the area of gateway monitoring and control.

Implementations of the authentication protocol specified in this document are likely to evolve in response to the particular security and privacy needs of its users. While, in general, the association between particular half-sessions of the authentication protocol and the described triplets of functions is specific to an implementation and beyond the scope of this document, the desire for immediate interoperability among initial implementations of this protocol is best served by the temporary adoption of a common authentication scheme. Accordingly, initial implementations will associate with every possible half-session a triplet of functions that realizes a trivial authentication mechanism:

(1) The authentication function is defined to have the value TRUE over the entire domain of authentication protocol messages.

(2) The message interpretation function is defined to be the identity function.

(3) The message representation function is defined to be the identity function.

Because this initial posture with respect to authentication is not likely to remain acceptable indefinitely, implementors are urged to adopt designs that isolate authentication mechanism as much as possible from other components of the implementation.

13. Appendix 5: Routing Information Propagation Variables

This section describes a set of related variables that characterize the sources and destinations of routing information propagated by various routing protocols. These variables have meaning only for those routing protocol implementations that afford greater flexibility in propagating routing information than is required by the various routing protocol specifications.

Each IP interface afforded by the configuration of the gateway over which routing information may propagate via a routing protocol 
(target interface) is named by a string of four octets that literally represents the IP address associated with said protocol interface.

Each IP protocol interface afforded by the configuration of the gateway over which routing information may arrive via any routing protocol (source interface) is named by a string of four octets that literally represents the IP address associated with said protocol interface.

Each routing protocol by which a gateway receives information that it uses to route IP traffic (source routing protocol) is named by a single-octet string according to the conventions set forth in Appendix 6 of this document.

Each routing protocol by which a gateway propagates routing information used by other hosts or gateways to route IP traffic (target routing protocol) is named by a single-octet string according to the conventions set forth in Appendix 6 of this document.

A variable such that the initial portion of its name is the concatenation of:

(1) the octet string represented symbolically as "_GW_pr_in_rif" and numerically as 01040104 followed by:

(2) the name of a target routing protocol followed by

(3) the name of a target interface followed by

(4) the name of a source routing protocol followed by

(5) the name of a source interface

has an integer value that characterizes the propagation of routing information between the sources and destinations of such information that are identified by the initial portion of that variable's name. A non-zero value for such a variable indicates that routing information received via the source routing protocol named by the fourth component of the variable name on the source interface named by its fifth component is propagated via the target routing protocol named by the second component of the variable name over the target interface named by its third component. A zero value for such a variable indicates that routing information received via the source routing protocol on the source interface identified in the variable name is NOT propagated via the target routing protocol over the target interface identified in the variable name. 
14. Appendix 6: Routing Protocol Representation

Numeric representations for routing protocols are presented below.

$\begin{array}{ll}\text { Value } & \text { Routing Protocol } \\ ==================== \\ 0 & \text { None }-- \text { Reserved } \\ 1 & \text { Berkeley RIP Version } 1 \\ 2 & \text { EGP } \\ 3 & \text { GGP } \\ 4 & \text { Hello } \\ 5 & \text { Other IGRP }\end{array}$

15. Appendix 7: Proteon p4200 Release 7.4 Variables

This section describes implementation-specific variables presented by the implementation of this protocol in Software Release 7.4 for the Proteon p4200 Internet Router. These variable definitions are subject to change without notice.

15.1. The Network Interface Variables

This section describes a related set of variables that represent attributes of a network interface in the Proteon p4200 Internet Router gateway. Each such network interface is uniquely named by an implementation-specific octet string of length 1 .

15.1.1. The Generic Network Interface Variables

This section describes a related set of variables that represent attributes common to all network interfaces in the Proteon p4200 Internet Router gateway. Each generic network interface of a p4200 configuration is uniquely named by the concatenation of the octet string represented symbolically as "_GW_impl_Proteon_p4200-R7.4_netif" and numerically as:

\section{$\begin{array}{lllll}01 & F F & 01 & 01 & 01\end{array}$}

followed by the name of said network interface as described above.

15.1.1.1. The Generic _ovfl-in Variable Class

A variable such that the initial portion of its name is the concatenation of the name for a generic network interface followed by the octet string represented symbolically as "_ovfl-in" and numerically as 01, has an integer value that represents the number of input packets dropped due to gateway congestion for the network interface identified by the initial portion of its name. 
15.1.1.2. The Generic _ovfl-out Variable Class

A variable such that the initial portion of its name is the concatenation of the name for a generic network interface followed by the octet string represented symbolically as "_ovfl-out" and numerically as 02, has an integer value that represents the number of output packets dropped due to gateway congestion for the network interface identified by the initial portion of its name.

15.1.1.3. The Generic_slftst-pass Variable Class A variable such that the initial portion of its name is the concatenation of the name for a generic network interface followed by the octet string represented symbolically as "_slftst-pass" and numerically as 03, has an integer value that represents the number of times the interface self-test procedure succeeded for the network interface identified by the initial portion of its name.

15.1.1.4. The Generic_slftst-fail Variable Class

A variable such that the initial portion of its name is the concatenation of the name for a generic network interface followed by the octet string represented symbolically as "_slftst-fail" and numerically as 04, has an integer value that represents the number of times the interface self-test procedure failed for the network interface identified by the initial portion of its name.

15.1.1.5. The Generic_maint-fail Variable Class

A variable such that the initial portion of its name is the concatenation of the name for a generic network interface followed by the octet string represented symbolically as "_maint-fail" and numerically as 06, has an integer value that represents the number of times the network maintenance procedure failed for the network interface identified by the initial portion of its name.

15.1.1.6. The Generic_csr Variable Class

A variable such that the initial portion of its name is the concatenation of the name for a generic network interface followed by the octet string represented symbolically as "_csr" and numerically as 07 , has an integer value that represents the internal address of the device CSR for the network interface identified by the initial portion of its name.

15.1.1.7. The Generic _vec Variable Class

A variable such that the initial portion of its name is the concatenation of the name for a generic network interface followed by the octet string represented symbolically as "_vec" and numerically

Davin, Case, Fedor and Schoffstall

[Page 26] 
as 08, has an integer value that identifies the device interrupt vector used by the network interface identified by the initial portion of its name.

\subsubsection{The ProNeT Network Interface Variables}

This section describes a related set of variables that represent attributes of a ProNET type network interface in the Proteon p4200 Internet Router gateway. Each network interface of a p4200 configuration that supports ProNeT media is uniquely named by the concatenation of the octet string represented symbolically as "_GW_impl_Proteon_p4200-R7.4_devpn" and numerically as:

$$
01 \text { FF } 010104
$$

followed by the name of said network interface as described above.

15.1.2.1. The Pronet_node-number Variable Class

A variable such that the initial portion of its name is the concatenation of the name for a ProNeT type network interface followed by the octet string represented symbolically as "_nodenumber" and numerically as 01, has an integer value that represents the ProNET node number associated with the network interface identified by the initial portion of its name.

\subsubsection{The ProNET_in-data-present Variable Class}

A variable such that the initial portion of its name is the concatenation of the name for a ProNET type network interface followed by the octet string represented symbolically as "_in-datapresent" and numerically as 02, has an integer value that represents the number of times that unread data was present in the input packet buffer for the network interface dentified by the initial portion of its name.

\subsubsection{The ProNET _in-overrun Variable Class}

A variable such that the initial portion of its name is the concatenation of the name for a ProNET type network interface followed by the octet string represented symbolically as "_inoverrun" and numerically as 03, has an integer value that represents the number of times that a packet copied from the ring exceeded the size of the packet input buffer on the network interface identified by the initial portion of its name. 


\subsubsection{The ProNET _in-odd-byte-cnt Variable Class}

A variable such that the initial portion of its name is the concatenation of the name for a ProNeT type network interface followed by the octet string represented symbolically as "_in-oddbyte-cnt" and numerically as 04, has an integer value that represents the number of times that a packet was received with an odd number of bytes on the network interface identified by the initial portion of its name.

15.1.2.5. The ProneT _in-parity-error Variable Class

A variable such that the initial portion of its name is the concatenation of the name for a ProNeT type network interface followed by the octet string represented symbolically as "_inparity-error" and numerically as 05, has an integer value that represents the number of times that a parity error was detected in a packet copied from the ring on the network interface identified by the initial portion of its name.

15.1.2.6. The ProNET _in-bad-format Variable Class

A variable such that the initial portion of its name is the concatenation of the name for a ProNET type network interface followed by the octet string represented symbolically as "_in-badformat" and numerically as 06, has an integer value that represents the number of times that a format error was detected in a packet copied from the ring on the network interface identified by the initial portion of its name.

15.1.2.7. The ProNET_not-in-ring Variable class

A variable such that the initial portion of its name is the concatenation of the name for a ProNET type network interface followed by the octet string represented symbolically as "_not-inring" and numerically as 07, has an integer value that represents the number of times that the ProNeT wire center relays were detected in an unenergized state for the network interface identified by the initial portion of its name.

\subsubsection{The ProNET _out-ring-inits Variable Class}

A variable such that the initial portion of its name is the concatenation of the name for a ProNeT type network interface followed by the octet string represented symbolically as "_out-ringinits" and numerically as 08, has an integer value that represents the number of times that ring initialization has been attempted on the network interface identified by the initial portion of its name. 


\subsubsection{The ProNET _out-bad-format Variable Class}

A variable such that the initial portion of its name is the concatenation of the name for a ProNeT type network interface followed by the octet string represented symbolically as "_out-badformat" and numerically as 09, has an integer value that represents the number of times that an improperly formatted packet was detected in the course of an output operation on the network interface identified by the initial portion of its name.

15.1.2.10. The Pronet_out-timeout Variable Class

A variable such that the initial portion of its name is the concatenation of the name for a ProNET type network interface followed by the octet string represented symbolically as "_outtimeout" and numerically as $0 \mathrm{~A}$, has an integer value that represents the number of times that an attempt to originate a message has been delayed by more than $700 \mathrm{~ms}$ on the network interface identified by the initial portion of its name.

\subsubsection{The Ethernet Network Interface Variables}

This section describes a related set of variables that represent attributes of an Ethernet type network interface in the Proteon p4200 Internet Router gateway. Each network interface of a p4200 configuration that supports Ethernet media is uniquely named by the concatenation of the octet string represented symbolically as "_GW_impl_Proteon_p4200-R7.4_dev-ie" and numerically as:

\section{FF 010103}

followed by the name of said network interface as described above.

15.1.3.1. The Ethernet _phys-addr Variable Class

A variable such that the initial portion of its name is the concatenation of the name for an Ethernet type network interface followed by the octet string represented symbolically as "_phys-addr" and numerically as 01 has an octet string value that literally represents the Ethernet station address associated with the network interface identified by the initial portion of its name.

\subsubsection{The Ethernet_input-ovfl Variable Class}

A variable such that the initial portion of its name is the concatenation of the name for an Ethernet type network interface followed by the octet string represented symbolically as "_inputovfl" and numerically as 02, has an integer value that represents the 
number of times the size of a received frame exceeded the maximum allowable for the network interface identified by the initial portion of its name.

15.1.3.3. The Ethernet _input-dropped Variable Class

A variable such that the initial portion of its name is the concatenation of the name for an Ethernet type network interface followed by the octet string representedo symbolically as "_inputdropped" and numerically as 03, has an integer value that represents the number of times the loss of one or more frames was detected on the network interface identified by the initial portion of its name.

15.1.3.4. The Ethernet_output-retry Variable Class

A variable such that the initial portion of its name is the concatenation of the name for an Ethernet type network interface followed by the octet string represented symbolically as "_outputretry" and numerically as 04, has an integer value that represents the number of output operations retried as the result of a transmission failure on the network interface identified by the initial portion of its name.

15.1.3.5. The Ethernet _output-fail Variable Class

A variable such that the initial portion of its name is the concatenation of the name for an Ethernet type network interface followed by the octet string represented symbolically as "_outputfail" and numerically as 05, has an integer value that represents the number of failed output operations detected on the network interface identified by the initial portion of its name.

15.1.3.6. The Ethernet_excess-coll Variable Class

A variable such that the initial portion of its name is the concatenation of the name for an Ethernet type network interface followed by the octet string represented symbolically as "_excesscoll" and numerically as 06, has an integer value that represents the number of times a transmit frame incurred 16 successive collisions when attempting media access via the network interface identified by the initial portion of its name.

\subsubsection{The Ethernet ffrag-rcvd Variable Class}

A variable such that the initial portion of its name is the concatenation of the name for an Ethernet type network interface followed by the octet string represented symbolically as "_frag-rcvd" and numerically as 07 , has an integer value that represents the 
number of collision fragments (i.e., "runt packets") that were received and filtered by the controller for the network interface identified by the initial portion of its name.

15.1.3.8. The Ethernet fframes-lost Variable Class

A variable such that the initial portion of its name is the concatenation of the name for an Ethernet type network interface followed by the octet string represented symbolically as "_frameslost" and numerically as 08, has an integer value that represents the number of frames not accepted by the Receive FIFO due to insufficient space for the network interface identified by the initial portion of its name.

15.1.3.9. The Ethernet_multicst-accept Variable Class

A variable such that the initial portion of its name is the concatenation of the name for an Ethernet type network interface followed by the octet string represented symbolically as "_multicstaccept" and numerically as 09, has an integer value that represents the number of frames received with a multicast-group destination address that matches one of those assigned to the controller for the network interface identified by the initial portion of said variable name.

15.1.3.10. The Ethernet_multicst-reject Variable class

A variable such that the initial portion of its name is the concatenation of the name for an Ethernet type network interface followed by the octet string represented symbolically as "_multicstreject" and numerically as $0 A$, has an integer value that represents the number of frames detected as having a multicast-group destination address that matches none of those assigned to the controller for the network interface identified by the initial portion of said variable name.

15.1.3.11. The Ethernet_crc-error Variable Class

A variable such that the initial portion of its name is the concatenation of the name for an Ethernet type network interface followed by the octet string represented symbolically as "_crc-error" and numerically as $O B$, has an integer value that represents the number of frames received with a CRC error on the network interface identified by the initial portion of its name.

15.1.3.12. The Ethernet _alignmnt-error Variable Class

A variable such that the initial portion of its name is the 
concatenation of the name for an Ethernet type network interface followed by the octet string represented symbolically as "_alignmnterror" and numerically as $0 \mathrm{C}$, has an integer value that represents the number of frames received with an alignment error on the network interface identified by the initial portion of its name. A received frame is said to have an alignment error if its received length is not an integral multiple of 8 bits.

15.1.3.13. The Ethernet_collisions Variable Class

A variable such that the initial portion of its name is the concatenation of the name for an Ethernet type network interface followed by the octet string represented symbolically as "_collisions" and numerically as OD, has an integer value that represents the number of collisions incurred during transmissions on the network interface identified by the initial portion of its name.

15.1.3.14. The Ethernet_out-of-window-coll Variable Class

A variable such that the initial portion of its name is the concatenation of the name for an Ethernet type network interface followed by the octet string represented symbolically as "_out-ofwindow-coll" and numerically as $0 \mathrm{E}$, has an integer value that represents the number of out-ofwindow collisions incurred during transmissions on the network interface identified by the initial portion of its name. Outof-window collisions are those occurring after the first 51.2 microseconds of slot time.

15.1.4. The Serial Network Interface Variables

This section describes a related set of variables that represent attributes of an serial line type network interface in the Proteon p4200 Internet Router gateway. Each network interface of a p4200 configuration that supports serial communications is uniquely named by the concatenation of the octet string represented symbolically as "_GW_impl_Proteon_p4200-R7.4_dev-sl" and numerically as:

01 FF 010105

followed by the name of said network interface as described above.

15.1.4.1. The Serial _tx-pkts Variable Class

A variable such that the initial portion of its name is the concatenation of the name for a serial line type network interface followed by the octet string represented symbolically as "_tx-pkts" and numerically as 01 , has an integer value that represents the number of packets transmitted on the network interface identified by

Davin, Case, Fedor and Schoffstall

[Page 32] 
the initial portion of its name.

15.1.4.2. The Serial _tx-framing-error Variable Class

A variable such that the initial portion of its name is the concatenation of the name for a serial line type network interface followed by the octet string represented symbolically as "_txframing-error" and numerically as 02, has an integer value that represents the number of transmission framing errors for the network interface identified by the initial portion of its name.

15.1.4.3. The Serial _tx-underrns Variable Class

A variable such that the initial portion of its name is the concatenation of the name for a serial line type network interface followed by the octet string represented symbolically as "_txunderrns" and numerically as 03, has an integer value that represents the number of transmission underrun errors for the network interface identified by the initial portion of its name.

15.1.4.4. The Serial_tx-no-dcd Variable Class

A variable such that the initial portion of its name is the concatenation of the name for a serial line type network interface followed by the octet string represented symbolically as "_tx-no-dcd" and numerically as 04 , has an integer value that represents the number of times transmission failed due to absence of the EIA Data Carrier Detect signal on the network interface identified by the initial portion of its name.

15.1.4.5. The Serial_tx-no-cts Variable Class

A variable such that the initial portion of its name is the concatenation of the name for a serial line type network interface followed by the octet string represented symbolically as "_tx-no-cts" and numerically as 05, has an integer value that represents the number of times transmission failed due to absence of the EIA Clear To Send signal on the network interface identified by the initial portion of its name.

\subsubsection{The Serial_tx-no-dsr Variable Class}

A variable such that the initial portion of its name is the concatenation of the name for a serial line type network interface followed by the octet string represented symbolically as "_tx-no-dsr" and numerically as 06 , has an integer value that represents the number of times transmission failed due to absence of the EIA Data Set Ready signal on the network interface identified by the initial 
portion of its name.

15.1.4.7. The Serial_rx-pkts Variable Class

A variable such that the initial portion of its name is the concatenation of the name for a serial line type network interface followed by the octet string represented symbolically as "_rx-pkts" and numerically as 07 , has an integer value that represents the number of packets received on the network interface identified by the initial portion of its name.

15.1.4.8. The Serial _rx-framing-err Variable Class

A variable such that the initial portion of its name is the concatenation of the name for a serial line type network interface followed by the octet string represented symbolically as "_rxframing-err" and numerically as 08, has an integer value that represents the number of receive framing errors on the network interface identified by the initial portion of its name.

15.1.4.9. The Serial _rx-overrns Variable Class

A variable such that the initial portion of its name is the concatenation of the name for a serial line type network interface followed by the octet string represented symbolically as "_rxoverrns" and numerically as 09, has an integer value that represents the number of receive overrun errors on the network interface identified by the initial portion of its name.

15.1.4.10. The Serial_rx-aborts Variable Class

A variable such that the initial portion of its name is the concatenation of the name for a serial line type network interface followed by the octet string represented symbolically as "_rx-aborts" and numerically as $0 \mathrm{~A}$, has an integer value that represents the number of aborted frames received on the network interface identified by the initial portion of its name.

15.1.4.11. The Serial_rx-crc-err Variable Class

A variable such that the initial portion of its name is the concatenation of the name for a serial line type network interface followed by the octet string represented symbolically as "_rx-crcerr" and numerically as $0 B$, has an integer value that represents the number of frames received with CRC errors on the network interface identified by the initial portion of its name.

Davin, Case, Fedor and Schoffstall

[Page 34] 
15.1.4.12. The Serial _rx-buf-ovfl Variable Class

A variable such that the initial portion of its name is the concatenation of the name for a serial line type network interface followed by the octet string represented symbolically as "_rx-bufovfl" and numerically as $0 \mathrm{C}$, has an integer value that represents the number of received frames whose size exceeded the maximum allowable on the network interface identified by the initial portion of its name.

15.1.4.13. The Serial_rx-buf-locked Variable Class

A variable such that the initial portion of its name is the concatenation of the name for a serial line type network interface followed by the octet string represented symbolically as "_rx-buflocked" and numerically as OD, has an integer value that represents the number of received frames lost for lack of an available buffer on the network interface identified by the initial portion of its name.

15.1.4.14. The Serial_rx-line-speed Variable Class

A variable such that the initial portion of its name is the concatenation of the name for a serial line type network interface followed by the octet string represented symbolically as "_rx-linespeed" and numerically as $0 \mathrm{E}$, has an integer value that represents an estimate of serial line bandwidth in bits per second for the network interface identified by the initial portion of its name. 